\title{
Laterality and Symmetry in Rat Olfactory Behavior and in Physiology of Olfactory Input
}

\author{
Kalyanasundaram Parthasarathy and Upinder S. Bhalla \\ National Centre for Biological Sciences, Tata Institute of Fundamental Research, Bangalore 560065, India
}

\begin{abstract}
Many species use bilateral sampling for odor-guided navigation. Bilateral localization strategies typically involve balanced and lateralized sensory input and early neuronal processing. For example, if gradient direction is estimated by differential sampling, then any asymmetry could bias the perceived direction. Subsequent neuronal processing can compensate for this asymmetry but requires the presence of mechanisms to track changes in asymmetry. A high degree of laterality is also important for differential sampling because spillover of signals will dilute the perceived odor gradient. In apparent contradiction to this model, both symmetry and laterality of nasal air flow have been reported to be incomplete in rats. Here, we measured symmetry and laterality in early olfactory processing in the rat. We first established behavioral readouts of precisely controlled bilateral odorant stimuli. We found that rats could rapidly and accurately report the direction of a wide range of odor gradients, presented in random sequence. We then showed that nasal air flow was symmetric over an entire day in awake rats. Furthermore, odor sampling from the two nostrils in the behavioral task was highly lateralized. This lateralization extended to the receptor epithelium responses as measured by electro-olfactograms. We finally observed strong lateralization of intrinsic signal responses from the glomerular layer of the olfactory bulb. We confirmed that a differential comparison of glomerular responses was sufficient to localize odorants. Together, these results suggest that the rat olfactory system is symmetric, with highly lateralized odor flow and neuronal responses. In combination, these attributes support odor localization by differential comparison.
\end{abstract}

\section{Introduction}

Rodents use odor information to navigate. Natural odors form a nonuniform, fluctuating concentration gradient, which can be estimated either by sequential sampling of different locations one after the other or by simultaneous sampling of two different locations in the environment (Vickers, 2000).

Bilateral sampling is important for depth perception in vision (Ohzawa et al., 1997) and sound localization in audition (Konishi, 2003). Similarly, bilateral odor sampling contributes significantly to odor localization in many species. This has been shown in fruit flies (Borst and Heisenberg, 1982; Duistermars et al., 2009; Gomez-Marin et al., 2010, 2011), sharks (Gardiner and Atema, 2010), rats (Rajan et al., 2006), and humans (von Bekesy, 1964; Porter et al., 2006). In principle, bilateral comparison of odor concentration provides instantaneous information about the odor gradient of the plume (Webster et al., 2001). Armed with this information the animal can track odors efficiently (Vickers,

Received April 9, 2012; revised Jan. 23, 2013; accepted Feb. 21, 2013.

Author contributions: K.P. and U.S.B. designed research; K.P. performed research; K.P. and U.S.B. analyzed data; K.P. and U.S.B. wrote the paper.

This work was supported by Grant BT/01/CEIB/09/III/03 from the Department of Biotechnology, India, to U.S.B. We thank Florin Albeanu and Ashesh K. Dhawale for their help with the intrinsic imaging setup, initial phases of recording, and analysis and Vivek Jayaraman for helpful comments and discussions on the project.

The authors declare no competing financial interests.

Correspondence should be addressed to Dr. Upinder S. Bhalla, National Centre for Biological Sciences, Tata Institute of Fundamental Research, Bellary Road, Bangalore 560065, India. E-mail: bhalla@ncbs.res.in.

DOI:10.1523/JNEUROSCI.1781-12.2013

Copyright $\odot 2013$ the authors $\quad 0270-6474 / 13 / 335750-11 \$ 15.00 / 0$
2000; Willis and Avondet, 2005; Porter et al., 2006; Steck et al., 2010; Khan et al., 2012).

Sensory asymmetry complicates the bilateral odor sampling strategy, as it introduces bias. This can be compensated by subsequent neural processing if the asymmetry is fixed, as in ear asymmetry in owls (Volman and Konishi, 1990). However, it has been reported that in many mammals there is a varying difference in the air flow rates between the two nostrils (Bojsen-Moller and Fahrenkrug, 1971; Hasegawa and Kern, 1977; Porter et al., 2005, 2006). In rats, the nasal cycle has been reported to have a period of 30-85 min (Bojsen-Moller and Fahrenkrug, 1971). It is therefore useful to ascertain whether additional neural mechanisms to compensate for asymmetry and the nasal cycle are indeed required in the rat olfactory pathway.

In addition to sensory equivalence, the lateralization of bilateral sampling is also important for sensitive differential comparison, as any spillover would weaken the odor gradient and hence diminish the differential signal. Here too, previous work has suggested that laterality may be incomplete because of odor flow across the nasal septum (Coppola et al., 1994). Furthermore, the proximity of the rat nostrils makes spillover seem likely, although there is evidence that the actual sampling is farther apart than the nostrils themselves (Wilson and Sullivan, 1999). Anatomically, the information flow in the olfactory circuitry is strictly lateralized as both the hemispheres do not have any cross-connection until the information reaches anterior olfactory nucleus (Bennett, 1968; Brunjes et al., 2005).

In the present study, we have used behavior and physiology to characterize the laterality and temporal stability of odor informa- 
tion representation in the olfactory receptors and bulb. Our results suggest that there is minimal sampling bias. Furthermore, we find there is very little cross-nostril signal at the input layer of the olfactory bulb (OB), either from sampling or from contralateral projections.

\section{Materials and Methods}

All experimental procedures were approved by the ethics committee of National Centre for Biological Sciences and in accordance with the guidelines of Government of India.

Animals. Six male Wistar rats, 3-4 months old, were used for the behavior experiment. Seven female Wistar rats, 4-5 months old, were used for the electro-olfactogram (EOG) recordings. Five female Wistar rats, 2-3 months old, were used for the optical intrinsic imaging experiment.

Female rats were used for the anesthetized preparations because of difficulties in maintaining male rats in the anesthetic plane for long periods $(\sim 6-9 \mathrm{~h})$ using a combination of ketamine $(100 \mathrm{mg} / \mathrm{kg})$ and xylazine $(10 \mathrm{mg} / \mathrm{kg})$. In previous work, we have shown that male and female rats do not significantly differ in their odor localization performances (Rajan et al., 2006). So, we do not expect any gender-based variation in the data.

Behavior setup. The setup used was similar to the ones used in an earlier study (Rajan et al., 2006). Briefly, the animal sat in a cubic box, $\sim 30 \mathrm{~cm}$ on each side. One side of the box housed a sniff port ( $15 \mathrm{~mm}$ diameter) in the center, and on either side there was a water delivery port $(20 \mathrm{~mm}$ diameter). Odor was delivered on each side through two polyurethane tubes, which were placed perpendicular to the rat's snout. IR beam interruption detection circuits were used to detect nose pokes and water port licks (see Fig. 1A). Custom-made electronic circuits and custom software were used to control the setup.

Odor stimulus delivery. A custom-built air dilution olfactometer was used to produce range of odor concentrations (0.03-3\%). The basic olfactometer design was described in detail previously (Rajan et al., 2006). Briefly, nitrogen was bubbled through glass bottles containing liquid odor. This odorized nitrogen $(0.0015-0.15 \mathrm{~L} / \mathrm{min})$ was injected into a continuous stream of clean air $(5 \mathrm{~L} / \mathrm{min})$ to obtain final odor concentration. In earlier experiments, a change of $0.05 \mathrm{~L} / \mathrm{min}$ in the total flow was shown not to provide behavioral cues (Rajan et al., 2006). In an extension of the original design, digital mass flow controllers (Celerity and Alicat Scientific) were used to regulate the nitrogen flows, which in turn decided the final odor concentrations.

We used two stimulus configurations: (1) unilateral stimulus-Odorized nitrogen was injected into the air stream of one side. The unilateral stimulus delivery olfactometer was used for the EOG experiment; and (2) bilateral stimulus - In this setup, each side had its own odor source and mass flow controllers. Odorized nitrogen was injected simultaneously into the air stream on both sides.

Calibration of olfactometer. The olfactometer was calibrated using Photo Ionization Detector (miniPID, Aurora Scientific). The probe was placed in front of the odor tubes perpendicular to the air stream, and data were acquired at $1 \mathrm{KHz}$. We obtained 15 samples of each of the following: isoamyl acetate odor concentrations (a, $0.5 \%$; b, 1\%; and c, 2\%) for both the left and right odor channels. The obtained average odor profiles of the left side were closely similar to that of the right side (see Fig. $1 C$ ). We used this odor profile to estimate the delay in the odor arrival to the rat resulting from the tubing, which was $\sim 70 \mathrm{~ms}$. The calibration was done only with the odor isoamyl acetate, as we used this odor for the behavioral experiments.

Odor localization task with bilateral odor cues. Three rats were first trained to locate an odor, isoamyl acetate, coming from either right or left at a single concentration (Rajan et al., 2006). After successful training on this odor localization task (task accuracy criterion $\geq 80 \%$ ), the rats were tested on two different conditions: (1) unilateral odor presentationodor comes from either left or right, the same as the training phase; and (2) bilateral odor presentation-odor comes from both sides at different concentrations. In both of these conditions, the rats had to determine which side had the higher concentration, to receive water rewards on that side (see Fig. $1 A, B$ ).

We used the following odor concentration stimulus pairs (left sideright side): $0-0.5,0-1,0.5-2,1-2,2-1,2-0.5,1-0$, and $0.5-0 \%$. The stimulus was randomly interleaved, and the rats' performance and sampling time were measured. Control trials with equal odor concentration on both sides (1-1\%) were introduced during one or two experimental sessions for each rat $(\sim 11 \%$ trials/session $)$. For these trials, the rats were rewarded on $50 \%$ of the trials, regardless of the odor direction or the response. Rats had access to water only during the behavior session ( $\sim 400$ trials; $45 \mathrm{~min}$ ), and ad libitum for $1.5 \mathrm{~d}$ over the weekend. The water spout was adjusted to deliver $\sim 24 \mu \mathrm{l}$ of water for each correct trial.

Response index (RI) calculation. RI is a measure of the performance of rats in the bilateral odor presentation experiment. The RI is defined as follows: RI = (left entry - right entry)/(left entry + right entry). Thus, a positive RI value indicates that most of the time the rat chose to go to the left side for reward and a negative RI indicates the converse.

Visualizing air flow pattern. To visualize the air flow pattern in our behavior setup, we used a planar laser-induced scattering technique. The air was seeded with liquid nitrogen fog. A green laser pointer $(<5 \mathrm{~mW})$ was projected through a cylindrical lens to form a sheet of light $\sim 2 \mathrm{~mm}$ thick. This sheet was used to illuminate the water particles. The light sheet was aligned parallel to the face of the behavior box and positioned as far from the box as the center of the odor delivery tubes. The illuminated particles were recorded at 25 frames per second, using a video camera (Sony Handycam DCR-SR300E, Sony). The images were obtained by averaging 30 frames.

Nasal air pressure recording. To verify the nasal air flow asymmetry between the nostrils, we used pressure sensors to record the nasal air pressure changes during respiration. The pressure readings act as a surrogate for flow rate measurements. Our implant procedures were similar to that of Wesson et al. (2009) with slight modifications. We implanted 11 naive rats with custom-made bilateral canula-pedestal assembly. The canulae were lowered into the small holes drilled on left and right sides, 1 $\mathrm{mm}$ anterior and $1 \mathrm{~mm}$ lateral to the midpoint of the nasal suture. The depth of the canulae was adjusted to obtain larger signal. The surgical anesthetic plane of the rats was maintained using halothane anesthesia, $4-5 \%$ for induction and $1-2 \%$ for maintenance. Postsurgery rats were orally administered the general analgesic Dolo SUS (paracetamol, 100 $\mathrm{mg} / \mathrm{kg}$, Micro Laboratories) for $5 \mathrm{~d}$. The nasal pressure recordings were done using pressure sensors (Freescale MPX10, 0-10 kPa). The signals were sampled at $1 \mathrm{kHz}$ and filtered between 0 and $470 \mathrm{~Hz}$ and, in some cases, an open filter (i.e., $0-9 \mathrm{kHz}$ ). The amplifier gain was 2000. Pressure sensor signals were continuously recorded for many hours with some gaps resulting from animal discomfort and technical issues, such as tube blockage. Recordings were for $24 \mathrm{~h}$ for 6 rats and $6-10 \mathrm{~h}$ for the remaining 5 rats.

Constructing right to left ratio profile of nasal pressure signals. We constructed right to left pressure signal ratio profile of the recording sessions to compare nasal pressure across the nostrils and to look for any periodicity in the signal. Before estimating the ratio of right to left pressure signals, we used an automated procedure to process the raw data to eliminate flat regions in recordings and technical issues with the signal, such as amplifier saturation. The flat regions were the result of occasional blockage of pressure sensor canulae.

We used custom MATLAB code to scan for regions where the signal amplitude was flat and close to baseline, in 2-s-long windows. These identified no-signal regions were removed from both the left and right datasets along with an additional $2 \mathrm{~s}$ following it. This additional $2 \mathrm{~s}$ typically showed intermediate levels of signal blockage. Both the left and right side nasal pressure data were processed independently. For some cases, we had gaps in the recordings (Rat 1, 10-30 min; Rats 2-4, 1-10 $\mathrm{min})$. We replaced these gaps with the mean of the signal for the entire recording session, as the subsequent analyses including Fourier transforms are insensitive to the mean. The DC offset was removed from the pressure signals for further analysis. For every minute of right samples versus left samples, a scatter plot was made. A regression fit was done for each such plot to estimate the slope, which is equal to the ratio right/left. The ratio was calculated for the data segments with at least 15,000 data 
points, which is equivalent to $15 \mathrm{~s}$ of data. In cases with $<15 \mathrm{~s}$ of data in any given minute of recording, we replaced the reading with the mean ratio for the entire session. This process gave us the time series of rightto-left ratio for the entire recording, sampled every minute. For the purpose of comparing nasal pressure signals of right and left sides, we constructed a coarse ratio profile for each recording session by taking the mean ratio over every $30 \mathrm{~min}$ window.

Spectrograms were computed using the MATLAB "spectrogram" function, and the grayscale range was adjusted for better visibility.

Periodicity analysis. We looked for periodicity in the ratio of right to left pressure signals, estimated for each 1 min segment using the rightversus-left scatter plots as above. We extracted the amplitude of the frequency components present in the ratio data using the Discrete Fourier Transformation analysis (MATLAB function "fft"). The amplitudes of the largest frequency peak were taken for further analysis.

EOG recordings and analysis. EOG recordings were done from xylazine/ketamine-anesthetized rats $(n=7)$. Four odors were used as stimuli: 1,4-cineole, amyl acetate, methyl amyl ketone, and isoamyl acetate (all odors from Sigma-Aldrich). We used the unilateral configuration of the olfactometer for the EOG recordings, as described above.

The recording conditions were as follows: A, Air from contralateral side and air + odor from ipsilateral side; and B, Air + odor from contralateral side and air from ipsilateral side.

Odor concentration was varied randomly, the following 5 concentrations were used: $0.03,0.1,0.3,1$, and $3 \%$. The stimulus protocol consisted of a $4 \mathrm{~s}$ odor stimulus and $6 \mathrm{~s}$ of intertrial interval with 15 repeats.

The onset of odor delivery was synchronized with the inspiration phase of respiration using custom built electronics.

For the analysis, we used the maximum EOG amplitude that occurred in the odor period averaged across the trials. This mean maximum EOG amplitude was normalized to the maximum amplitude for that odor within the recording session.

Optical intrinsic imaging. A custom-assembled setup was used to image from the olfactory bulbs of anesthetized rats. The bulb surface was illuminated using an infrared LED ( $\sim 780 \mathrm{~nm}$, Roithner Laser Technik). Images were acquired at a frame rate of $31 \mathrm{~Hz}$ and with 14 bit resolution using a CCD camera (Andor iXon DV-887 BI). The camera was attached to a reversed lens macro assembly (Nikon Nikkor lenses, focal length: 35 and $50 \mathrm{~mm} ; 1.4 \times$ magnification), and the pixel size was $\sim 11 \mu \mathrm{m}$. We used the following four odors to stimulate olfactory receptors: isoamyl acetate, valeraldehyde, ethyl tiglate, and 1,4-cineole (all odors from Sigma-Aldrich). The odor concentrations used and the odor delivery tube arrangements were identical to the bilateral odor cue behavior experiment. A single trial consisted of $15 \mathrm{~s}$ of air followed by $15 \mathrm{~s}$ of odorized air. Trials were repeated with an intertrial interval of $45 \mathrm{~s}$. Each stimulus was repeated seven times and was interleaved with other odors and intensity combinations.

Image analysis. We followed the same method as described previously (Meister and Bonhoeffer, 2001; Soucy et al., 2009) to extract odor response information from the recorded frames. A ratio image was composed for each trial by dividing the average odor period (15 s) image by the average air period ( $15 \mathrm{~s}$ ) image. Then an average ratio image for each stimulus combination was generated from the repeats. The averaged image was further high pass filtered to get rid of low spatial frequency noise. Filtering was done by subtracting the low pass filtered image from the averaged image The low pass filtered image was computed by convolving the image with a Gaussian spatial kernel $(S D=330 \mu \mathrm{m})$. After filtering, previously inconspicuous spots become prominent. These discrete spots are the result of odor stimulated absorption change in the glomerulus (Belluscio and Katz, 2001; Meister and Bonhoeffer, 2001; Wachowiak and Cohen, 2003; Soucy et al., 2009). Each spot was fitted with a two-dimensional Gaussian to estimate the spot's response amplitude. The estimated amplitude was used to represent that spot's response to all the odors and intensity used.

Individual glomerulus analysis. The idea of this analysis was to determine whether we could identify the high odor concentration side using a differential comparison of intrinsic signal responses on the two sides. We selected only those glomeruli (spots), which were responsive to the given odor. For simplicity, we used the response amplitude $R$ obtained from the raw $\Delta R / R$ values unlike the previous analysis which used fitted values. We normalized $\mathrm{R}$ to a maximum of 1 for each glomerulus. We generated the dose-response curves for each glomerulus for the specified odor and used these to obtain the odor concentration for half-maximal response $H R$, for each glomerulus. We then estimated the differential signal $D$ for each trial as follows:

$$
\mathrm{D}=\sum_{\text {Left glomeruli }} \mathrm{HR}-\sum_{\text {Right glomeruli }} \mathrm{HR}
$$

When $D$ is $<0$, the right side is stronger, and vice versa. This value of $D$ could be further scaled to achieve a maximum response index (defined above) of 1 for our concentration range. For simplicity, we did not do this as it would involve more complicated weights for each glomerulus and would have to be reestimated for each odor and each experiment. Given our concentration ranges, $D$ is mathematically smaller than 1 for all our concentration combinations.

The number of samples per odor was as follows: isoamyl acetate $(n=11), 1,4$-cineole $(n=21)$, valeraldehyde $(n=28)$, and ethyl tiglate $(n=35)$.

\section{Results}

In this study, we first characterize the robustness, sensitivity, and timing of stereo odor localization behavior in the presence of a range of odor gradients between nostrils. We then show that sampling asymmetry is minimal in awake behaving rats and that rats are able to localize the direction of an odor gradient even when odors are present at a range of concentrations on both sides, in a random order. We use EOG recordings and intrinsic signal imaging to show that physiological responses are ipsilateral both at the receptor and bulb level. We finally show that intrinsic signal responses are sufficient to account for behavioral decisions, on a trial-by-trial basis.

\section{Rats can identify the high intensity side when odor is present bilaterally}

To study how rats use bilateral odor sampling information, we used a behavioral task based on standard operant conditions. The task required rats to select the higher odor concentration side to get a water reward. We considered two metrics of behavior performance: accuracy and sampling time.

Three rats were trained on an odor localization task (Fig. $1 A, B$; see Materials and Methods), where odor was presented unilaterally at fixed concentration, $1 \%$. Once the rats reached the criterion accuracy $(80 \%)$ on this task, they were tested with the following set of left-right odor concentration pairs, all reported as percentage of saturation: $0-0.5,0-1,0.5-2,1-2,1-1,2-1$, $2-0.5,1-0$, and $0.5-0 \%$. We calibrated the olfactometer to check that these concentration gradients were accurate and symmetric (Fig. 1C). One of the stimulus pairs (1-1) presented equal amount of odor on both sides and was used as a control (probe) trial. Probe trials were delivered only in one or two of the sessions for each rat. These probe trials accounted for $11 \%$ of total trials and were rewarded randomly either on left or right with the probability of 0.5 . The objective of this probe trial was to test the response to symmetric input. In the following sections, the odor gradient is defined as the arithmetic difference between the odor concentrations on each side (left-right), with a positive gradient having a higher concentration on the left.

\section{Performance improves with steeper odor gradients}

The stimulus pairs were randomly presented and rats performed $\sim 400-500$ trials per session. We found that rats reliably selected the high odor concentration side (Fig. 2A). The influence of odor intensity difference in the side choice made by the rats was further analyzed by computing a response index RI (RI = (left entry - 
A

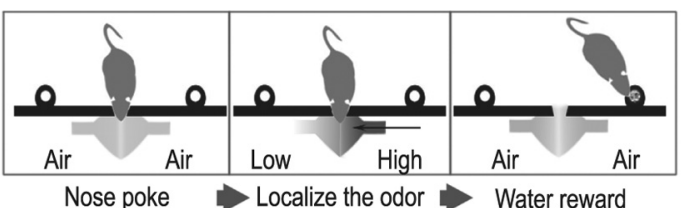

B

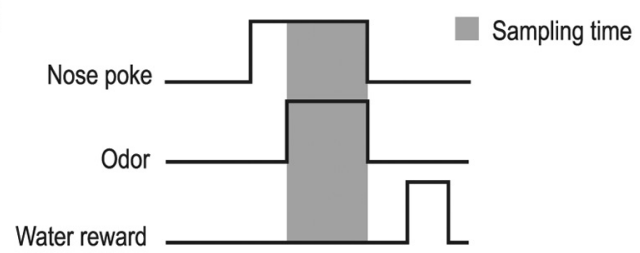

C

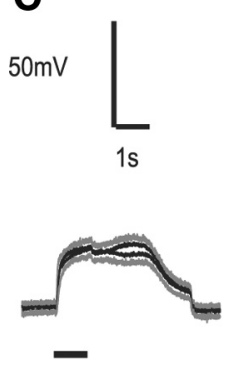

$0.5 \%$

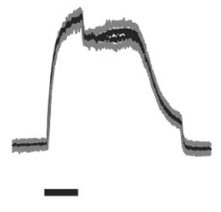

$1 \%$

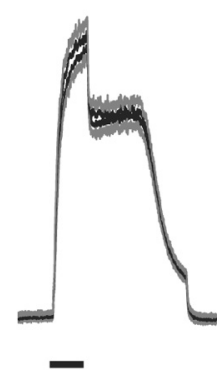

$2 \%$

Figure 1. $A, 0$ dor localization task. The sequence is as follows: nose poke until odor comes on, localize the odor, and go to respective side for water reward. $\boldsymbol{B}$, Behavior task event sequence. Sampling time is estimated as the time from odor onset to nose withdrawal. C, Calibration of odor stimulus, at $0.5 \%, 1 \%$, and $2 \%$ saturation. Traces are PID readings at the outlet of the olfactometer, averaged $>15$ presentations (black traces). The initial part of the curve (black bar) is the odor sampling time; the later part is the purge that occurs after nose withdrawal. The gray traces above and below the black traces indicate the SD from the upper and lower signal, respectively. No bias is apparent for $0.5 \%$ and $1 \%$ concentrations, and there is a small difference for the $2 \%$ concentration, which is within one SD.

A

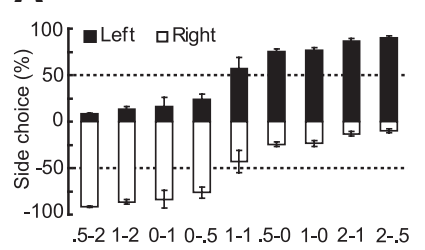

B

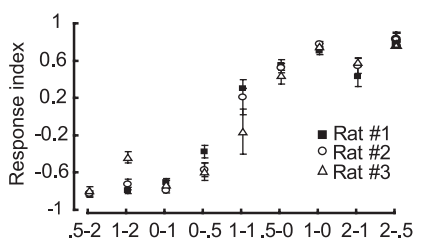

Concentration combination ( $L-R \%$ )

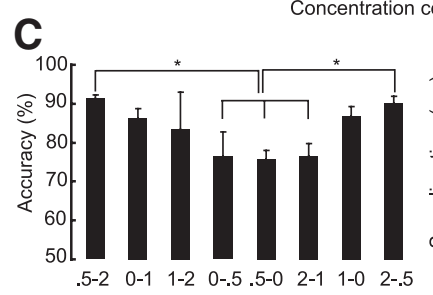

D

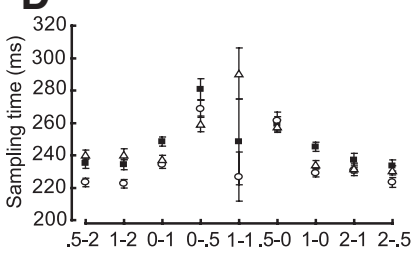

Concentration combination ( $\mathrm{L}-\mathrm{R} \%$ )

Figure 2. Rats do bilateral comparison to localize odor; $n=3$. $A$, Side choice made by the rats in the gradient experiment. $\boldsymbol{B}$, Performance as a function of concentration combination categories. The performance is measured in terms of Rl, where $\mathrm{Rl}=$ (left entry - right entry)/ (left entry + right entry). Positive values indicate more left side choices, and negative values indicate more right side choices. Side choice depended on the steepness of the odor gradient. For equal concentration trials, rats performed around chance level. $C$, Accuracy for different concentration combination categories. ${ }^{*} p=0.0014$ (one-way ANOVA, $F_{(7,16)}=6.08$; Tukey post hoc comparison of the categories). $\boldsymbol{D}$, Sampling time as a function of concentration combination. Rats take $220-300 \mathrm{~ms}$ to make their side choices. Error goes up for the equal concentration trials. $B, D$, Error bars indicate SEM. $A, C$, Error bars indicate SD. right entry)/(left entry + right entry); see Materials and Methods). We observed that the RI swung from $\sim-0.8$ to +0.8 as the odor gradient increased (Fig. 2B). Specifically, the RI was larger for larger gradients and was close to zero for the probe trials which had a zero gradient.

As described in the Materials and Methods, the response accuracy was defined as the percentage of time that the rat correctly selected the higher concentration side. Even though the overall performance stayed $\sim 80 \%$ accuracy, there was some dissimilarity in performance between the gradient combinations. The localization accuracy was best with steeper gradients. Accuracy was degraded at less steep gradients and was significantly worse for the lowest gradient $(0-0.5$ and vice versa) compared with the highest $(0.5-2)$ (Fig. $2 B, C$; one-way ANOVA, $F_{(7,16)}=6.08,{ }^{\star} p=$ 0.0014 ; Tukey post hoc comparison of the categories). Interestingly, the highest performance case (0.5-2) had odor on both sides, and this was significantly better (by the above test) than the unilateral cases $(0-0.5$ and $0.5-0)$ which had odor on only one side. Thus, absolute odor concentration rather than concentration ratio appears to be important for gradient discrimination.

\section{Symmetric input results in chance performance}

A key prediction from the hypothesis of a differential stereo strategy, using symmetric sensitivity of detection on each side is that a zero gradient with nonzero odor concentrations on either side should have no side bias. As described above, we conducted probe trials during which rats were exposed to equal amount of odor on both left and right. The side choices for these trials fell in the chance level regimen, $40-60 \%$ choices between the sides (Fig. $2 A, B)$. We view this result as behavioral support for the hypothesis that rats use "differential comparison" mechanisms to localize odor. In the sections below, we further characterize the sampling strategies and air flow patterns that are other essential components of this hypothesis.

Sampling time as a function of the steepness of odor gradient We found that the rats took $200-320 \mathrm{~ms}$ to localize the highconcentration odor arrival side (Fig. 2D). We have subtracted the tubing delay of $70 \mathrm{~ms}$ from the sampling time. The sampling time was smaller with steeper gradients and higher at less steep gradients. We find relatively little effect over our concentration ranges, although there was a (not significant) suggestion of slower responses at lower concentrations. The variance in the sampling times of equal concentration trials (probe trials) was large, possibly because of fewer trials (45-100), whereas other categories had a range of 300-1000 trials.

Overall, performance accuracy and sampling time had only a slight dependence on gradient. The results so far suggest that rats are indeed sensitive to odor gradients and are not biased in their side choices when the stimulus is symmetric.

\section{The nasal air pressure is comparable between the nostrils}

We next sought to find out the effects of bilateral "sensor asymmetry" on a differential comparison strategy. It has been reported that rats have a nasal cycle similar to humans. Air flow in the two nostrils is not equal and this asymmetry alternates over a time scale of 30-85 min in anesthetized rats (Bojsen-Moller and Fahrenkrug, 1971). It is not clear whether this also happens in awake rats.

To estimate input asymmetry and the nasal cycle, we used pressure readings in awake rats as a surrogate for flow rate measurements. Naive rats were implanted with bilateral canulae for pressure recordings (Fig. 3A,B; see Materials and Methods). 
During the recording period, rats were in their home cage and freely moving. These recordings spanned $24 \mathrm{~h}$ (Fig. $3 C$; see Materials and Methods). The pressure signals on the two sides were tightly correlated, and their relative amplitude showed little change over the entire $24 \mathrm{~h}$ cycle. In all but one of the recordings (Rat 11), the leftright signals were balanced within $10 \%$. In Rat 11 , the signals were consistently biased to the left, which we speculate is a systematic change resulting from canula placement. To more closely examine the responses at different times of day, we examined half-hour sections of the recordings at 1:00 A.M. and 2:30 P.M. Even at this finer sampling, the plots of leftversus-right signals lay closely on a straight, 45-degree line (Fig. 3D,E). This was despite large differences in respiration patterning during these half-hour samples (Fig. $3 F, G$ ).

We also checked for the cyclic changes in the left-versus-right values over the entire recorded period of time at a finer time scale, one minute (Fig. 4A; Rat 11). The frequency components were extracted from the ratio data using a Fourier transform (Fig. 4 B; see Materials and Methods). The amplitudes of the obtained frequency components were all smaller than that the SDs of their respective right/ left ratio data (Fig. 4C). In other words, any periodic component in the signal was smaller than the noise distribution and considerably smaller than the two SD criterion for significance. For comparison, the amplitude of a pure sine wave is $\sqrt{ } 2$ larger than its SD (root mean square). The distribution of the largest periodic components across the recording sessions ranged from 8 to $520 \mathrm{~min}$ (Fig. 4D), without any clear peak. Furthermore, this range was larger than the previously reported range of $30-85 \mathrm{~min}$ (Bojsen-Moller and Fahrenkrug, 1971).

Together, these results show that air flow in the nostrils of the awake rat is symmetric and that this symmetry is maintained over the entire day.

\section{The two nostrils sample different parts of the odor gradient}

The observed sampling time in the behavior task ranged from 200 to $320 \mathrm{~ms}$, which could accommodate more than one sniff (Rajan et al., 2006; Kepecs et al., 2007). This may have provided the rat with enough time to sample the odor by moving its head from side to side to sample different parts of the odor stream. This strategy has been shown to be effective in odor localization in Drosophila larvae (Louis et al., 2008; Gomez-Marin et al., 2011). To address this issue, we tracked the position of the nostrils of three rats during the task. For this purpose, some of the behavior sessions were video recorded and the nostril position was marked on a frame-by-frame basis (Fig. 5A; see Materials and Methods). D-G, Data from Rat 9.
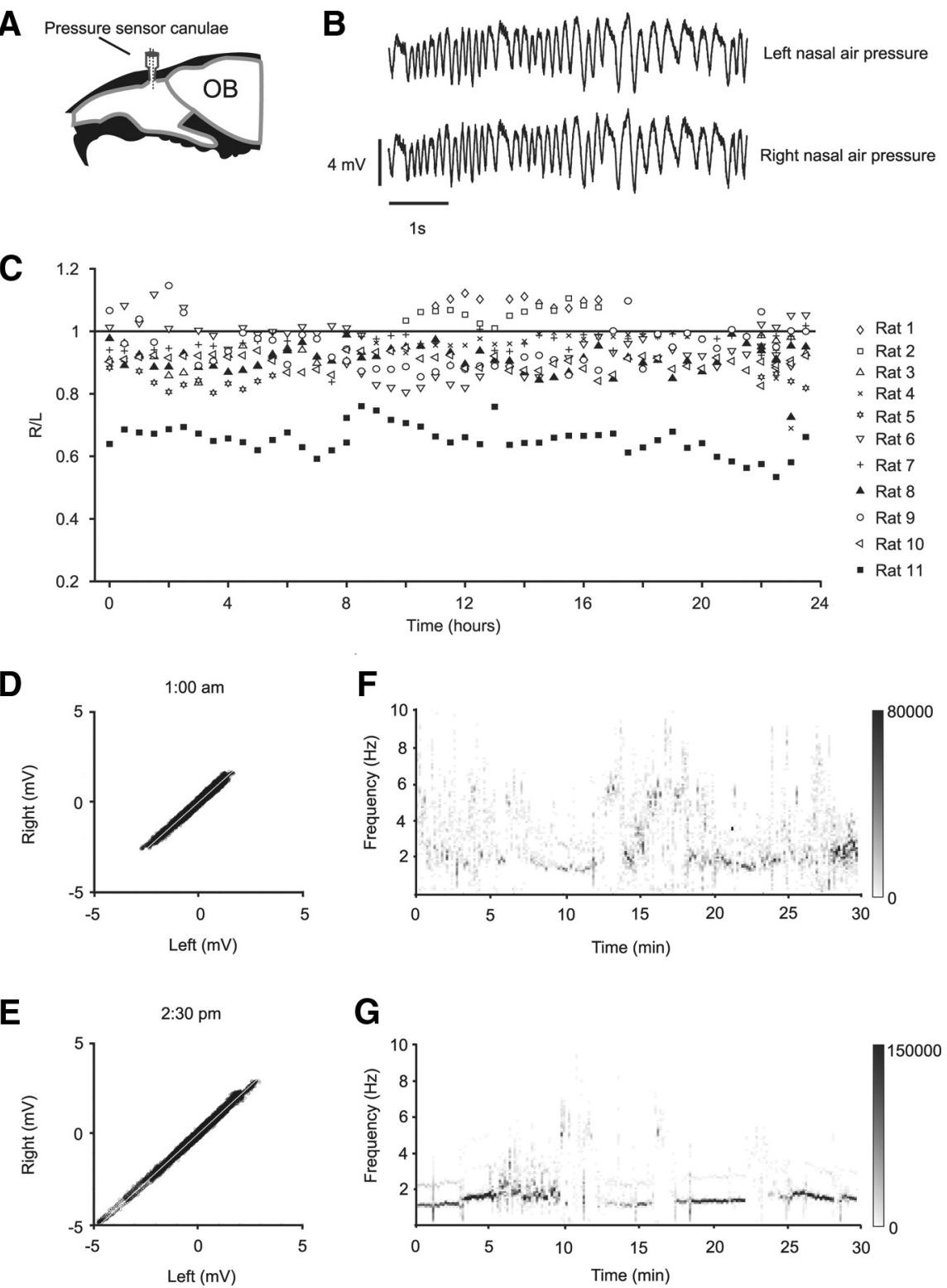

Figure 3. The nasal air pressures in the two nostrils are comparable. $\boldsymbol{A}$, Diagram shows implant location. $\boldsymbol{B}$, Traces of nasal air one with the exception of Rat 11. D, Scatter plots of right versus left samples for a half-hour period at 1:00 A.M. E, Scatte Spectrogram of left channel for same data as in $\boldsymbol{E} . \boldsymbol{F}, \mathbf{G}$, Gray scale bar represents power value of spectrum (arbitrary units).

Nostril position tracking was done for at least 100 trials. During these trials, the performance of the rats remained $>70 \%$. In all three rats, the position of the nostril formed two clusters with little or no overlap (Fig. 5A). To further investigate the head movement during trials, we looked at the nostril movement trajectory within a given trial (Fig. $5 B$ ). This showed that rats' odor sampling was predominantly in the vertical plane. To quantify this observation, we calculated the difference between the consecutive frames of a trial, in both the vertical ( $y$-axis) and horizontal ( $x$-axis) planes. This analysis showed that the nostril movement was substantially restricted to vertical plane ( $y$-axis) (Fig. 5C).

A possible confound in the above result is that the air flow patterns themselves may be asymmetric or variable, leading to 
A

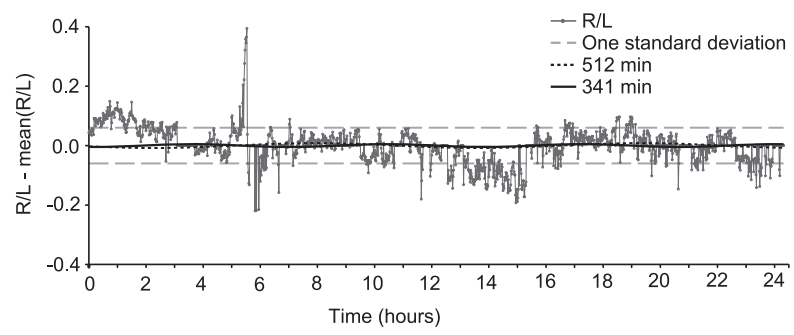

B

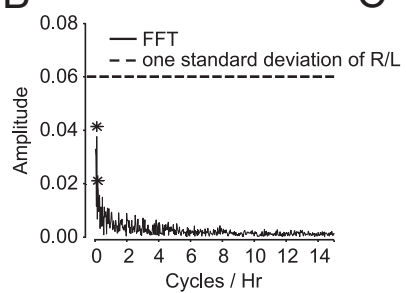

D

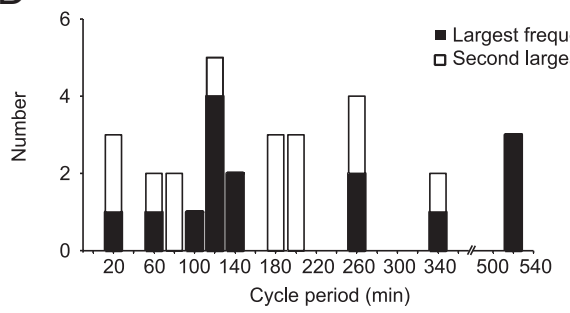

Figure 4. Cyclical changes in the nasal air pressure are smaller than signal noise. $A$, Ratio of right to left signals measured using a 1 min window, over the $24 \mathrm{~h}$ cycle. Data from Rat 11. Gray hyphenated lines indicate one SD above and below the mean. The flat regions in the plot (solid gray line) are segments where the recordings were interrupted (see Materials and Methods). The overlaid sine waves are the first two largest frequency components identified by the Discrete Fourier Transformation analysis (see Materials and Methods). B, Fast Fourier Transformation (FFT) amplitude spectrum of slope values. *The largest two frequency components. $C$, Scatter plot of amplitude of largest frequency component against SD, for all nasal pressure recordings. Open circles represent daytime recordings; filled circles, night recordings; gray circles, day and night recordings. The amplitude of largest frequency component was smaller than one SD of the signal (45-degree line) in all cases. D, Distribution of largest frequency components. Filled bars represent the largest frequencies; open bars, second largest frequencies.

differences in sampling even if the nostril positions did not overlap. We therefore also analyzed the air flow pattern in the setup (Fig. 5D; see Materials and Methods). As described in Materials and Methods, our behavior setup has a constant flow $(5 \mathrm{~L} / \mathrm{m})$ of air from both left and right odor tubes. The flow on one of the sides was visualized in the presence of contralateral air flow (Fig. $5 D$, left) and in the absence of contralateral air flow (Fig. 5D, right). The two conditions were strikingly different. In the case of balanced flow, the spread of the ipsilateral air stream was stopped by the opposing contralateral air stream close to the center of the nose poke ring. This dividing line corresponds well with the arrangement of nostril position clusters lying on either side of the center of the nose poke ring.

Thus, we find that rats can accurately localize odors even when their nostril positions are constrained so that they do not sequentially sample different parts of the odor gradient.

\section{Odor sampling is lateralized}

The above results from nostril tracking and air flow analysis suggest that the bilateral odor stimulus is lateralized, which in turn, could result in lateralized sampling by each nostril. To further support this, we measured the degree of spillover in our experimental design by carrying out EOG recordings from anesthetized
A
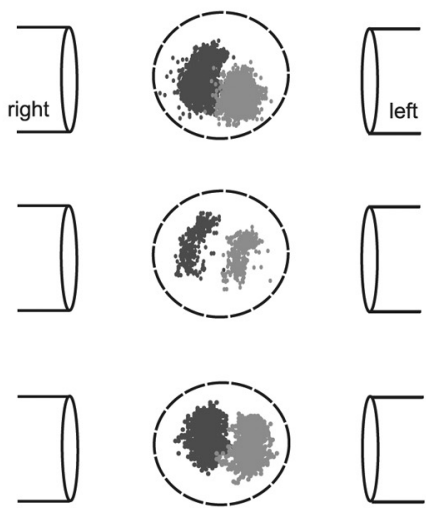

right nostril position

left nostril position
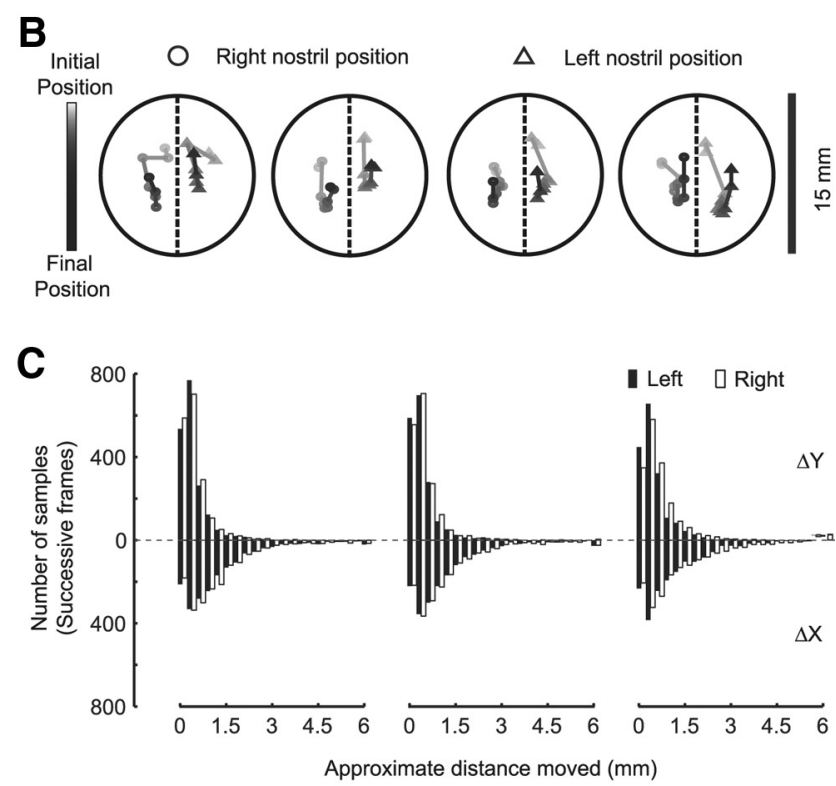

D
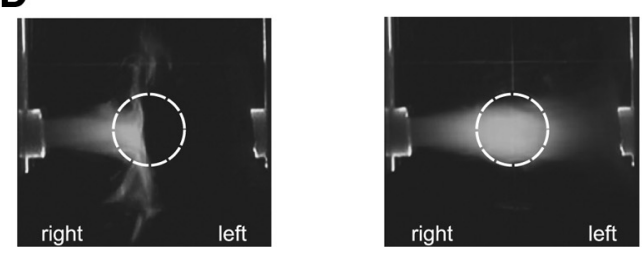

Figure 5. Rats sample distinct portions of the odor stimulus with their two nostrils. $\boldsymbol{A}$, Left (light gray) and right (dark gray) nostril position distribution during individual trials for odor localization behavior. Data from three rats. $\boldsymbol{B}$, Left and right nostril position trajectory of four trails of Rat 3 ( $\boldsymbol{A}$, Bottom). $\boldsymbol{C}$, Histogram showing the distribution of nostril's movement in both horizontal plane ( $\Delta \mathrm{X}$, below zero line) and vertical plane ( $\Delta \mathrm{Y}$, above zero line). The black bar represents left nostril movement distribution, and the empty bar indicates right nostril movement distribution. D, Air flow pattern with (left) and without (right) contralateral air stream. Flow was visualized using liquid nitrogen fog on the right channel.

rats. We delivered unilateral odorant stimuli and the apparatus was identical to the one used in the behavioral experiments. The experiment was performed under two conditions: with and without a contralateral air stream. For analysis, trials were sorted based on whether the odor was delivered on the same side as the recording electrode or the contralateral side.

We found that, in the presence of counterbalancing air stream, the EOG response scaled with odor concentration only 
A
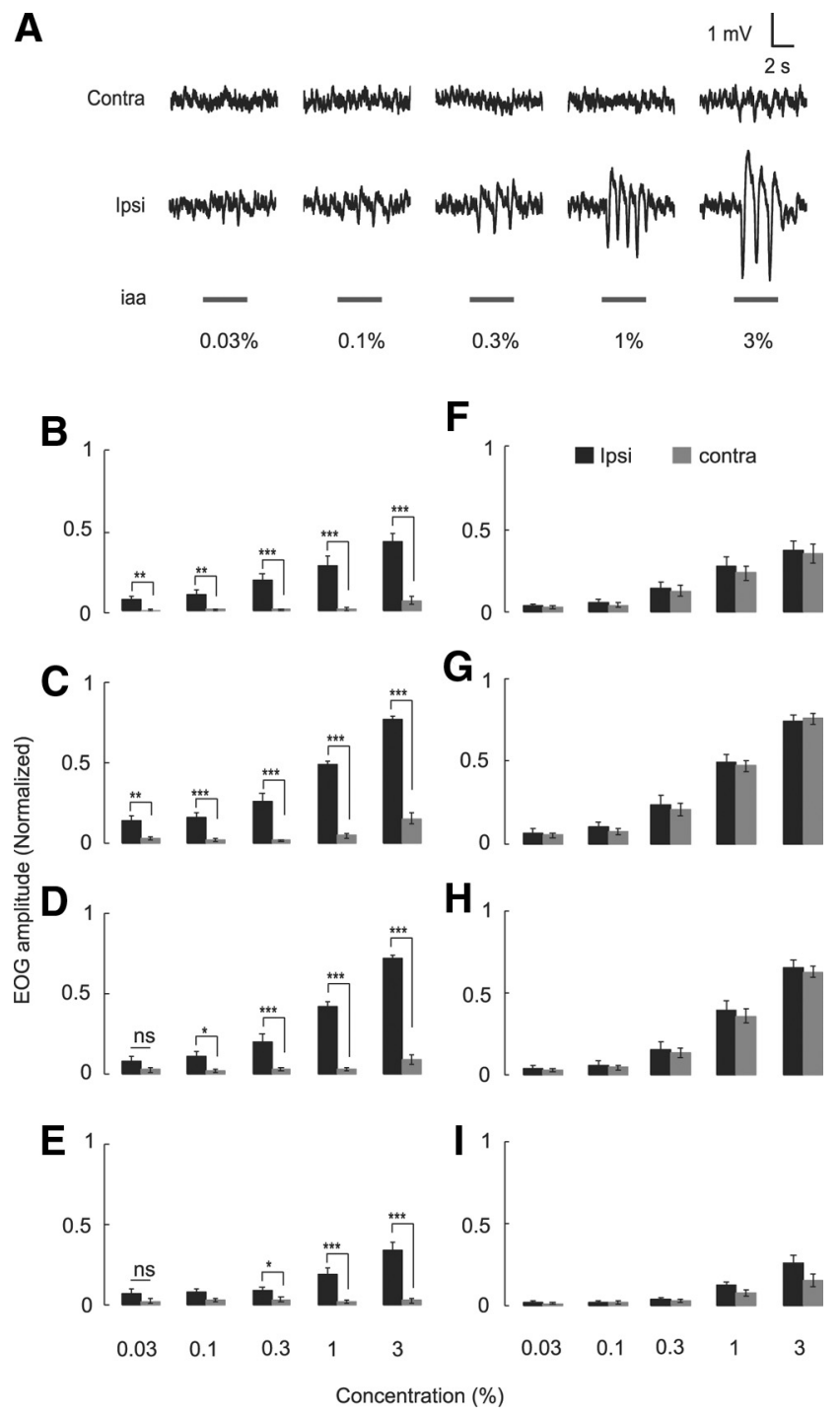

Figure 6. Odor does not spill over to the contralateral side. $A$, EOG signal recorded from both left and right nostrils for different concentrations of the odor isoamyl acetate (data from Rat 090408). $\boldsymbol{B}-\boldsymbol{I}$, Bar graphs showing normalized mean maximum EOG amplitude (odor-air) at five concentrations; $n=7$. Black bars indicate that the odor was delivered from the ipsilateral (Ipsi) side, and gray bars indicate that the odor was delivered from the contralateral side. $\boldsymbol{B}-\boldsymbol{E}$, No response from the contralateral side, when there was counterbalancing air stream on the contralateral side. ${ }^{*} p<0.05$, ipsilateral versus contralateral response pairs ( $t$ test). ${ }^{* *} p<0.01$, ipsilateral versus contralateral response pairs ( $t$ test). ${ }^{* * *} p<0.001$, ipsilateral versus contralateral response pairs ( $t$ test). ns, Not significant $(p>0.05)$. $F-I$, Response when there was no counterbalancing air on the contralateral side. For all plots, ipsilateral and contralateral response pairs ( $p>0.05, t$ test). Odor identities are as follows: $\boldsymbol{B}, \boldsymbol{F}, 1,4-$ Cineole. $\boldsymbol{C}, \boldsymbol{G}$, Isoamyl acetate (iaa). $\boldsymbol{D}, \boldsymbol{H}$, Amyl acetate. $\boldsymbol{E}, \boldsymbol{I}$, Methyl amyl ketone. Error bars indicate SEM.

on the ipsilateral side, whereas it remained at or near baseline on the contralateral side to the recording electrode (Fig. $6 A-E ; t$ test between ipsilateral and contralateral response pairs: ${ }^{\star} p<0.05$, ${ }^{* *} p<0.01,{ }^{* * *} p<0.001$, and not significant, $p>0.05$ ). The only case where there was detectable spillover was for isoamyl acetate and amyl acetate at an odor concentration of 3\%. When there was no contralateral air stream, the EOG response scaled comparably with both ipsilateral and contralateral stimuli (Fig. $6 F-I ; t$ test between ipsilateral and contralateral response pairs, $p>0.05$ for all the plots). This set of experiments shows that odor sampling is highly lateralized at the receptor level.
Ipsilateral responses in the glomeruli are not affected by contralateral input

To follow up our analysis of olfactory response lateralization for bilateral inputs, we used optical intrinsic imaging to study responses to bilateral stimuli in the glomerular layer of the olfactory bulb. The objectives of this experiment were to determine the representation of bilateral odor information at the level of olfactory input and to assess the influence of contralateral odor stimuli on the response to ipsilateral odor stimuli (Fig. 7A).

The bilateral stimulus pairs were the same as the behavior experiment, that is: $0-0.5,0-1,0.5-2,1-2,1-1,2-1,2-0.5,1-0$, and $0.5-0 \%$ of saturation.

To compare the activity between the sides, we needed to capture each OB's activity levels in a single value. For this purpose, we used principal component analysis to reduce dimensions (see Materials and Methods). The resulting principal component score represents the activity of each OB. The trials were sorted based on the ipsilateral odor intensity, in ascending order for the purpose of visualization. The glomerular activity pattern was unique for each odor (Fig. 7B), similar to earlier studies (Belluscio and Katz, 2001; Meister and Bonhoeffer, 2001; Wachowiak and Cohen, 2003; Soucy et al., 2009). The level of glomerular activity increased with increasing odor intensity (Fig. $7 B-D$ ). These results are in accordance with previous findings (Belluscio and Katz, 2001; Wachowiak and Cohen, 2003; Soucy et al., 2009). We did not observe any significant activity on the contralateral side for the unilateral odor presentation trials (Fig. $7 E ; t$ test, $\left.{ }^{* * *} p<0.001\right)$.

Although there was no response to contralateral odor stimuli during the unilateral odor presentation trials, we asked whether contralateral odor stimuli can modulate the response to an ipsilateral odor stimulus by presenting odor stimuli at different concentrations on both sides. The trials were grouped within the same ipsilateral odor intensity, and responses across different contralateral concentrations were compared for each group. If there was any influence of contralateral stimulus on ipsilateral odor response, this should have resulted in a systematic variation in the ipsilateral side odor response. We did not observe any significant difference in the ipsilateral activity within the groups (Fig. $7 F$; within the group, two-way unbalanced ANOVA, $F_{(3,263)}=$ $1.01, p=0.3903$; between the groups, $F_{(3,263)}=521.13, p \ll$ 0.001; Tukey post hoc comparison of ipsilateral odor response). This result suggests that the contralateral odorant input has no effect on the response at the glomerular level of the ipsilateral olfactory bulb.

\section{The bilateral glomerular response predicts the behavior}

We finally asked whether the glomerular intrinisic signal responses carried sufficient information to predict behavioral odor localization using a simple differential comparison. This test was facilitated by the use of identical odor gradient combinations in the behavior and intrinsic signal recordings.

It has been suggested that different glomeruli have different odor sensitivity (Meister and Bonhoeffer, 2001; Wachowiak and Cohen, 2001, 2003; Spors et al., 2006). To confirm this for our odor panel, we looked at individual glomerular responses as a function of ipsilateral odor concentration. In the overall population, we observed at least three kinds of glomeruli based on the dynamic range of their response to different concentrations of the given odor. Illustrative cases are shown in Figure 8A. We used the glomerulus half-maximal response $(H R)$ concentration for categorization (see Materials and Methods). Category $A$ has a satu- 
A

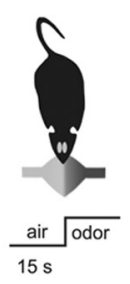

B
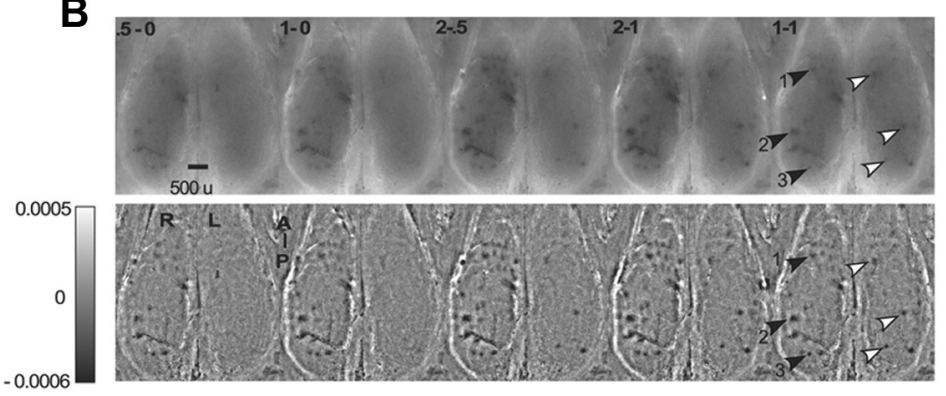

C

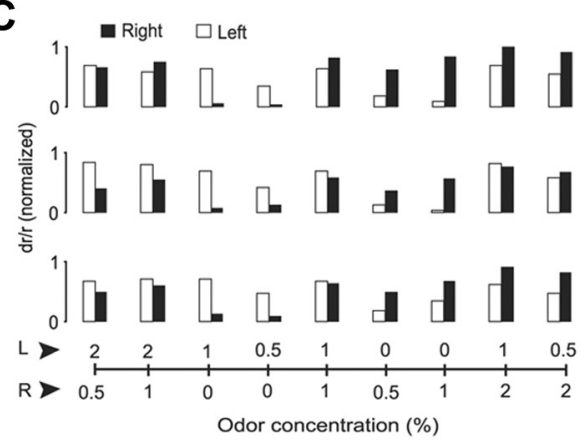

D

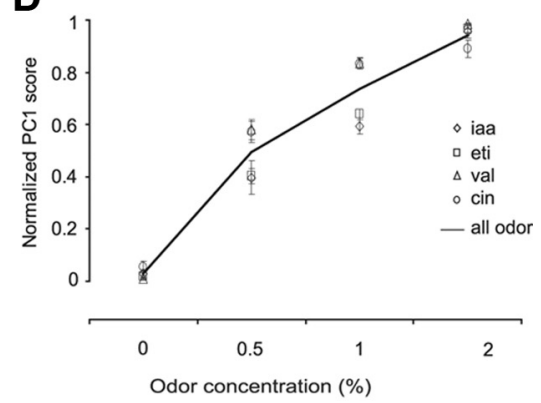

E

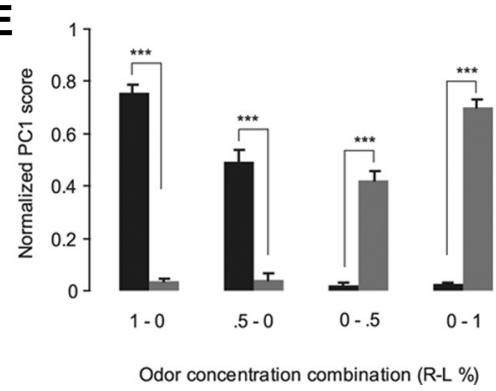

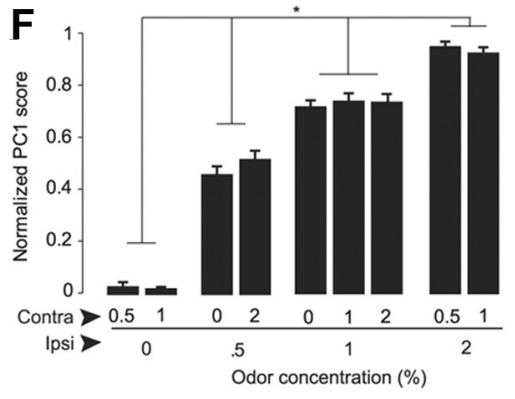

Figure 7. Ipsilateral response is not affected by contralateral stimuli. $\boldsymbol{A}$, Experimental schematic. $\boldsymbol{B}$, Illustrative intrinsic imaging from one of the datasets showing response of six glomeruli for the odor ethyl tiglate for 5 different odor gradients. Top row, Result of the ratio of the air period and odor period. Bottom row, Images were obtained by filtering out the low-frequency components from the ratio image. $\boldsymbol{C}$, Bar graphs showing intrinsic imaging response for six glomeruli to the odor ethyl tiglate, indicated by arrowheads in panel $\boldsymbol{B}$, three on each side (left, open bars; right, filled bars). The values on the $y$-axis is inverted for the plotting purpose. $\boldsymbol{D}$, Glomerular response as a function of odor concentration on ipsilateral side. Solid line represents the averaged concentration response profile. Individual data points mark the response for individual odors. Error bars indicate SEM. iaa, Isoamyl acetate; eti, ethyl tiglate; val, valeraldehyde; cin, 1,4-cineole. $\boldsymbol{E}$, Comparing ipsilateral versus contralateral responses for the unilateral odor trials. In all cases, the ipsilateral response is very different from contralateral: ${ }^{* * *} p<0.001$ (t test). $\boldsymbol{F}$, Ipsilateral odor response is not affected by contralateral odor stimuli. The combination categories are grouped based on their ipsilateral odor concentration. The categories within the groups are not significantly different from each other, but across the group they are significantly different from each other (two-way unbalanced ANOVA: within the group, $F_{(3,263)}=1.01, p=0.3903$; between groups, $F_{(3,263)}=521.13$, ${ }^{*} p<0.001$; Tukey post hoc comparison of ipsilateral odor response). rats, for a total of 95 samples. In each rat, we identified a subset of glomeruli that were activated for each odor. There was more than one active glomeruli for all odors except for 1,4-cineole, which triggered a response in only a single dorsal glomerulus. However, even this single glomerulus was sufficient to localize the odor to the left or right (Fig. $8 D$ ).

We compared $D$ for the entire set of trials with the behavioral response curve (Fig. 8C). Here, the error bars represent $\mathrm{SD}$, to facilitate comparison of distributions between the two cases. There was an excellent match for ipsilateral concentrations up to $1 \%$ (correlation coefficients; $p<0.0001, r=0.9705)$. Above that, the estimate of $D$ fell toward the zero-gradient mid-line. We interpret this as resulting from saturation of the response at $\sim 1 \%$ for most glomeruli (Fig. 8A,B). All four odors exhibited the same response pattern (Fig. $8 D$ ). How might the animal detect steeper gradients? From the distribution of glomeruli in Fig. $7 B$, it is clear that the simple measure of $D$ is biased toward glomeruli that saturate at lower concentrations. A more sophisticated estimate might give a greater weight to the less sensitive glomeruli, and indeed neural mechanisms in the bulb and piriform are known to tune responsiveness depending on context (Kay and Laurent, 1999; Wilson and Sullivan, 2011). We conclude that a simple differential comparison of glomerular responses carries enough information to account for the behavioral ability of rats to discriminate gradients.

\section{Discussion}

In this study, we have characterized the complementary attributes of symmetry and laterality of information flow through the early olfactory system. We suggest that together these constitute a parsimonious basis for the nervous system to perform differential comparisons for odor localization. rating response to all concentrations tested $(H R<0.25, n=111)$; $B$ responds to all the given concentrations in a linear fashion (HR $<0.75, n=35)$; and $C$ peaks for the highest concentration ( $H R<1.5, n=27$ ) (Fig. 8B). Based on this range of sensitivities, we computed the differential signal $D$ for each trial as described in Materials and Methods:

$$
\mathrm{D}=\sum_{\text {Left glomeruli }} \mathrm{HR}-\sum_{\text {Right glomeruli }} \mathrm{HR}
$$

Where $H$ is the concentration for half-maximal response for the glomerulus and $R$ is the actual measured response at the specified concentration. When $D$ is below zero, the right side is stronger, and vice versa.

We estimated $D$ for all 9 concentration combinations, for 6 or 7 intrinsic signal measurements each for four odors recorded in 5

\section{Bilateral balance}

Bilateral odor sampling is thought to improve odor localization by detecting odor gradients through differential comparisons between responses in the two nostrils. This is, in principle, a singlesniff strategy. This idea is complicated by possible asymmetry at various stages of olfaction. Asymmetry spoils the differential comparison strategy because it introduces a bias in perceived odor gradients. For example, a spatially uniform odor concentration would always be classified as a gradient. Given the subtlety and transient nature of natural odor directional cues and the narrow spacing between nostrils, even a small asymmetry would be prone to cause errors. This problem worsens if the asymmetry itself varies, as the sensitivity would then require continuous recalibration. Although such mechanisms are known to exist, as 

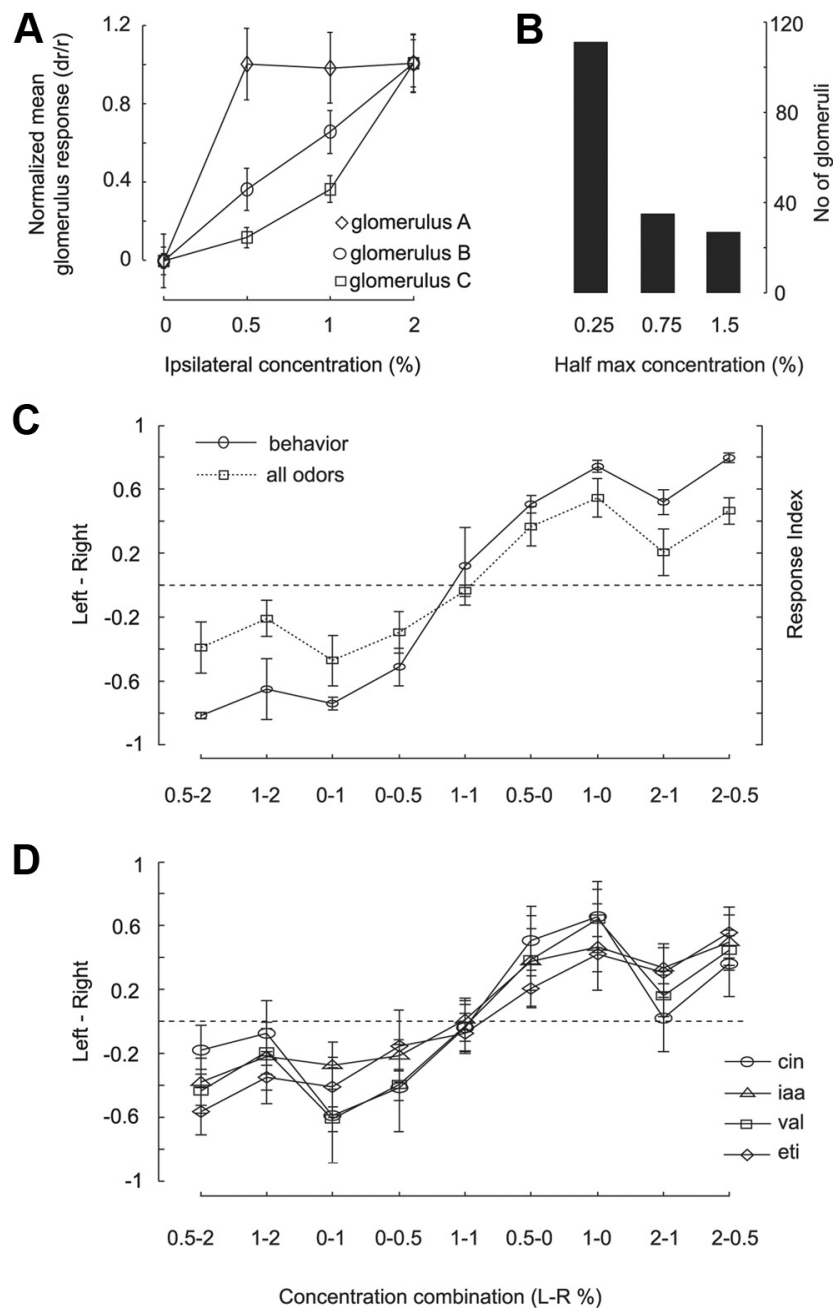

Figure 8. Bilateral glomerular response predicts behavior. $\boldsymbol{A}$, Example dose-response curves for glomerular activity as a function of odor concentration from one of the imaging sessions. $\boldsymbol{B}$, The distribution of glomeruli based on their half-maximum response concentration. C, Response index from behavior (solid line) compared with the differential comparison index based on glomerular intrinsic signals (hyphenated line). The intrinsic signal curve is a average of all four odors: cin, 1,4-cineole; iaa, isoamyl acetate; val, valeraldehyde; eti, ethyl tiglate. Error bar indicates SD of the mean. The $y$-axis scaling for the differential index for glomerular has not been normalized (see Materials and Methods). $\boldsymbol{D}$, Individual odors all exhibit similar differential comparison curves. Error bars indicate SD to facilitate comparison of distributions.

discussed below, we suggest that they are not predominant in the rat. A simpler assumption is that the sensitivity of the olfactory pathway is precisely balanced. This eliminates the need for a dynamic recalibration mechanism but imposes its own strict requirements on multiple levels of the olfactory pathway. Specifically, nasal air flow, olfactory receptors, and projections to the bulb would have to be well balanced and stable over time. In the current study, we have shown that all these are true in the rat. Behaviorally, one test of this hypothesis is if equal, nonzero concentrations at each nostril consistently lead to chance performance in reporting gradients, even if the recent history of stimulation includes random gradients and concentrations. This too is what we observe.

Natural odor stimuli typically involve a single odor source with odorant arriving at both nostrils in plumes, where each nostril samples from spatially separate regions. Our segregated dual odor source apparatus was designed to create a precisely controlled gradient, rather than recapitulate the more complex nat- ural air flow. However, we think that our results do address the underlying nasal physiology and neural mechanisms, given our finding that the two odor streams are highly lateralized once in the nose. There is indeed the caveat that sampling odor plumes may result in complex temporal structure of the odorant stimulus, which the current study does not address. Specifically, previous studies have shown that timing cues are also good for odor localization (Rajan et al., 2006), and in sharks such timing cues may be more salient than intensity differences (Gardiner and Atema, 2010).

It will be interesting to consider the question of symmetry versus calibration in other organisms in which nasal air flow has been reported to vary. Several careful studies have been performed in anesthetized large mammals, such as dogs (Lung and Wang, 1989) cats (Bamford and Eccles, 1982), and pigs (Eccles and Maynard, 1975; Eccles, 1978). These studies have used pressure sensors and other measurements, have all established cyclicity in left and right air flow, and have led to proposals of autonomic nervous system control of these cycles. Studies in the anesthetized rat have used condensation as an indirect measure of lateralized air flow (Bojsen-Moller and Fahrenkrug, 1971). Our study, in contrast to this, is in awake rats and uses pressure sensors. We propose that our finding of stable symmetry is more likely to be physiologically and behaviorally relevant for the rat.

Humans are also known to have cycles of left versus right air flow (Hasegawa and Kern, 1977; Porter et al., 2005, 2006). In human subjects performing an odor localization task, there was a significant difference between the peak sniff amplitude measured between the two nostrils (Porter et al., 2005). However, the sniff volume was not significantly different. Despite this difference in the amplitude, Porter et al. (2005) found no correlation between the nasal air flow asymmetry and odor localization performance of the subjects. They also addressed the concern about the possible odor percept difference between the nostrils resulting from the air flow asymmetry. By taking hints from the subjects after experimental debriefings about the strategy for localization, they suggested that it was less likely that the subjects used the percept difference to localize odor. One possibility is simply that humans are not as sensitive to small gradients, so minor asymmetries do not matter to them. Alternatively, subjects could recalibrate sensitivity to account for nasal air flow changes. This is especially likely because the reported time scale of nasal air flow changes $(\sim 2 \mathrm{~h})$ is much longer than those of the localization task (seconds).

Odor gradient detection need not depend on comparison of symmetric sensors (Bell and Tobin, 1982). Caenorhabditis elegans, for example, is thought to combine asymmetric receptor pairs with sequential sampling to perform chemotaxis (Sengupta, 2007). In Drosophila, asymmetric inhibition of a phermomonesensitive glomerulus leads to differences in response time depending on odor direction (Agarwal and Isacoff, 2011). This has been suggested to work with a winner-take-all circuit to report odor direction. We suggest that strategies that rely on asymmetric responses in the olfactory pathway are likely to be less robust in classifying unpredictable odor gradients with unpredictable concentrations, as present in nature and also in the current study. Furthermore, all our results point to rather well-balanced leftright sensitivity, in the early olfactory pathway in the rat.

\section{Lateralization}

Where does differential comparison begin? Bilaterally responsive neurons have been reported from the olfactory bulb (Rajan et al., 
2006). The same study also showed that these bilateral inputs are likely to be olfactory, rather than trigeminal, by including an odorant (phenethyl alcohol), which has been shown not to stimulate the trigeminal nerve (Silver and Moulton, 1982). Thus, we think that the relevant differential comparison pathway is olfactory, not trigeminal. In the olfactory pathway, the first interhemispherical connection is brought by the anterior olfactory nucleus $(\mathrm{AON})$, which relays information between the right and left olfactory bulbs via the anterior limb of the anterior commissure (AC) (Bennett, 1968; McBride and Slotnick, 1997; Brunjes et al., 2005; Kikuta et al., 2008). In support of this view, a recent study showed that neurons in the AON pars externa can detect the odor arrival side by modulating its firing rate in proportion to the odor intensity (Kikuta et al., 2010). An earlier study claimed that each nostril could access odor sampled by its counterpart through retronasal and internasal routes (Coppola et al., 1994). Our EOG experiments largely showed no odor spill over response in the contralateral side, although a small amount of spillover response was observed at high concentration (3\%) for the odors isoamyl acetate and amyl acetate. However, we find that the intrinsic signal responses of individual glomeruli are entirely ipsilaterally driven. In principle, one might expect there to be a contralateral influence on mitral/tufted cell dendritic tufts, and on the activity of periglomerular cells. Our intrinsic signal readings did not detect any such influence.

Our study shows that, consistent with the simplest theories for differential comparison, the rat olfactory system ensures clean lateralization right from the sampling stage through to the input layer of the olfactory bulb.

\section{Temporal information versus glomerular averaging in classification of odor gradients}

Rats and mice need only one or two sniffs to recognize odors in discrimination tasks (Uchida and Mainen, 2003; Abraham et al., 2004; Rinberg et al., 2006). Clearly, the same detailed temporal information is available for odor localization because the current and previous studies show that rats can localize an odor in one or two sniffs (Rajan et al., 2006). Having shown this, it is intriguing that our study shows that it is possible to extract directional information from the first-order, temporally averaged response represented by intrinsic signal activity in the glomerular layer. With the caveat that our intrinsic signal readings are themselves averaged $>15 \mathrm{~s}$, we find that the distribution of direction selectivity from single glomerular readings is consistent with the behavioral response selectivity. We therefore suggest that a differential comparison of total firing rate, or number of spikes per sniff, of receptor neurons is a good enough directional cue for first-order odor direction discrimination. Natural odor plumes in turbulent conditions may elicit higher-order temporal information in the receptor spike train that could ride upon this basic mechanism.

\section{References}

Abraham NM, Spors H, Carleton A, Margrie TW, Kuner T, Schaefer AT (2004) Maintaining accuracy at the expense of speed: stimulus similarity defines odor discrimination time in mice. Neuron 44:865-876. CrossRef Medline

Agarwal G, Isacoff E (2011) Specializations of a pheromonal glomerulus in the Drosophila olfactory system. J Neurophysiol 105:1711-1721. CrossRef Medline

Bamford OS, Eccles R (1982) The central reciprocal control of nasal vasomotor oscillations. Pflugers Arch 394:139-143. CrossRef Medline

Bell WJ, Tobin TR (1982) Chemo-orientation. Biol Rev 57:219-260. CrossRef
Belluscio L, Katz LC (2001) Symmetry, stereotypy, and topography of odorant representations in mouse olfactory bulbs. J Neurosci 21:21132122. Medline

Bennett MH (1968) The role of the anterior limb of the anterior commissure in olfaction. Physiol Behav 3:507-515. CrossRef

Bojsen-Moller F, Fahrenkrug J (1971) Nasal swell-bodies and cyclic changes in the air passage of the rat and rabbit nose. J Anat 110:25-37. Medline

Borst A, Heisenberg M (1982) Osmotropotaxis in Drosophila melanogaster. J Comp Physiol 147:479-484. CrossRef

Brunjes PC, Illig KR, Meyer EA (2005) A field guide to the anterior olfactory nucleus (cortex). Brain Res Brain Res Rev 50:305-335. CrossRef Medline

Coppola DM, Coltrane JA, Arsov I (1994) Retronasal or internasal olfaction can mediate odor-guided behaviors in newborn mice. Physiol Behav 56: 729-736. CrossRef Medline

Duistermars BJ, Chow DM, Frye MA (2009) Flies require bilateral sensory input to track odor gradients in flight. Curr Biol 19:1301-1307. CrossRef Medline

Eccles R (1978) The domestic pig as an experimental animal for studies on the nasal cycle. Acta Otolaryngol 85:431-436. CrossRef Medline

Eccles R, Maynard RL (1975) Proceedings: Studies on the nasal cycle in the immobilized pig. J Physiol 247:1P. Medline

Gardiner JM, Atema J (2010) The function of bilateral odor arrival time differences in olfactory orientation of sharks. Curr Biol 20:1187-1191. CrossRef Medline

Gomez-Marin A, Duistermars BJ, Frye MA, Louis M (2010) Mechanisms of odor-tracking: multiple sensors for enhanced perception and behavior. Front Cell Neurosci 4:6. CrossRef Medline

Gomez-Marin A, Stephens GJ, Louis M (2011) Active sampling and decision making in Drosophila chemotaxis. Nat Commun 2:441. CrossRef Medline

Hasegawa M, Kern EB (1977) The human nasal cycle. Mayo Clin Proc 52: 28-34. Medline

Kay LM, Laurent G (1999) Odor- and context-dependent modulation of mitral cell activity in behaving rats. Nat Neurosci 2:1003-1009. CrossRef Medline

Kepecs A, Uchida N, Mainen ZF (2007) Rapid and precise control of sniffing during olfactory discrimination in rats. J Neurophysiol 98:205-213. CrossRef Medline

Khan AG, Sarangi M, Bhalla US (2012) Rats track odour trails accurately using a multi-layered strategy with near-optimal sampling. Nat Commun 3:703. CrossRef Medline

Kikuta S, Kashiwadani H, Mori K (2008) Compensatory rapid switching of binasal inputs in the olfactory cortex. J Neurosci 28:11989-11997. CrossRef Medline

Kikuta S, Sato K, Kashiwadani H, Tsunoda K, Yamasoba T, Mori K (2010) Neurons in the anterior olfactory nucleus pars externa detect right or left localization of odor sources. Proc Natl Acad Sci U S A 107:12363-12368. CrossRef Medline

Konishi M (2003) Coding of auditory space. Annu Rev Neurosci 26:31-55. CrossRef Medline

Louis M, Piccinotti S, Vosshall LB (2008) High-resolution measurement of odor-driven behavior in Drosophila larvae. J Vis Exp 3:11. CrossRef Medline

Lung MA, Wang JC (1989) Autonomic nervous control of nasal vasculature and air flow resistance in the anaesthetized dog. J Physiol 419:121-139. Medline

McBride SA, Slotnick B (1997) The olfactory thalamocortical system and odor reversal learning examined using an asymmetrical lesion paradigm in rats. Behav Neurosci 111:1273-1284. CrossRef Medline

Meister M, Bonhoeffer T (2001) Tuning and topography in an odor map on the rat olfactory bulb. J Neurosci 21:1351-1360. Medline

Ohzawa I, DeAngelis GC, Freeman RD (1997) The neural coding of stereoscopic depth. Neuroreport 8:iii-xii. CrossRef Medline

Porter J, Anand T, Johnson B, Khan RM, Sobel N (2005) Brain mechanisms for extracting spatial information from smell. Neuron 47:581-592. CrossRef Medline

Porter J, Craven B, Khan RM, Chang SJ, Kang I, Judkewicz B, Volpe J, Settles G, Sobel N (2007) Mechanisms of scent-tracking in humans. Nat Neurosci 10:27-29. CrossRef Medline

Rajan R, Clement JP, Bhalla US (2006) Rats smell in stereo. Science 311: 666-670. CrossRef Medline 
Rinberg D, Koulakov A, Gelperin A (2006) Speed-accuracy tradeoff in olfaction. Neuron 51:351-358. CrossRef Medline

Sengupta P (2007) Generation and modulation of chemosensory behaviors in C. elegans. Pflugers Arch 454:721-734. CrossRef Medline

Silver WL, Moulton DG (1982) Chemosensitivity of rat nasal trigeminal receptors. Physiol Behav 28:927-931. CrossRef Medline

Soucy ER, Albeanu DF, Fantana AL, Murthy VN, Meister M (2009) Precision and diversity in an odor map on the olfactory bulb. Nat Neurosci 12:210-220. CrossRef Medline

Spors H, Wachowiak M, Cohen LB, Friedrich RW (2006) Temporal dynamics and latency patterns of receptor neuron input to the olfactory bulb. J Neurosci 26:1247-1259. CrossRef Medline

Steck K, Knaden M, Hansson BS (2010) Do desert ants smell the scenery in stereo? Anim Behav 79:939-945. CrossRef

Uchida N, Mainen ZF (2003) Speed and accuracy of olfactory discrimination in the rat. Nat Neurosci 6:1224-1229. CrossRef Medline

Vickers NJ (2000) Mechanisms of animal navigation in odor plumes. Biol Bull 198:203-212. CrossRef Medline

Volman SF, Konishi M (1990) Comparative physiology of sound localization in four species of owls. Brain Behav Evol 36:196-215. CrossRef Medline

von Bekesy G (1964) Olfactory analogue to directional hearing. J Appl Physiol 19:369-373. Medline
Wachowiak M, Cohen LB (2001) Representation of odorants by receptor neuron input to the mouse olfactory bulb. Neuron 32:723-735. CrossRef Medline

Wachowiak M, Cohen LB (2003) Correspondence between odorantevoked patterns of receptor neuron input and intrinsic optical signals in the mouse olfactory bulb. J Neurophysiol 89:1623-1639. CrossRef Medline

Webster DR, Rahman S, Dasi LP (2001) On the usefulness of bilateral comparison to tracking turbulent chemical odor plumes. Limnology Oceanography 46:1048-1053. CrossRef

Wesson DW, Verhagen JV, Wachowiak M (2009) Why sniff fast? The relationship between sniff frequency, odor discrimination, and receptor neuron activation in the rat. J Neurophysiol 101:1089-1102. CrossRef Medline

Willis MA, Avondet JL (2005) Odor-modulated orientation in walking male cockroaches Periplaneta americana, and the effects of odor plumes of different structure. J Exp Biol 208:721-735. CrossRef Medline

Wilson DA, Sullivan RM (1999) Respiratory air flow pattern at the rat's snout and an hypothesis regarding its role in olfaction. Physiol Behav 66:41-44. CrossRef Medline

Wilson DA, Sullivan RM (2011) Cortical processing of odor objects. Neuron 72:506-519. CrossRef Medline 